\title{
Identification of the Predicted 5s-4 $f$ Level Crossing Optical Lines with Applications to Metrology and Searches for the Variation of Fundamental Constants
}

\author{
A. Windberger, ${ }^{1,}$ J. R. Crespo López-Urrutia, ${ }^{1, \dagger}$ H. Bekker, ${ }^{1}$ N. S. Oreshkina, ${ }^{1}$ J. C. Berengut, ${ }^{2}$ V. Bock, ${ }^{1}$ \\ A. Borschevsky, ${ }^{3}$ V. A. Dzuba, ${ }^{2}$ E. Eliav, ${ }^{4}$ Z. Harman, ${ }^{1}$ U. Kaldor, ${ }^{4}$ S. Kaul,${ }^{1}$ U. I. Safronova, ${ }^{5}$ \\ V. V. Flambaum, ${ }^{2}$ C. H. Keitel, ${ }^{1}$ P. O. Schmidt, ${ }^{6,7}$ J. Ullrich, ${ }^{1,6}$ and O. O. Versolato, \\ ${ }^{1}$ Max-Planck-Institut für Kernphysik, Saupfercheckweg 1, 69117 Heidelberg, Germany \\ ${ }^{2}$ School of Physics, University of New South Wales, Sydney, New South Wales 2052, Australia \\ ${ }^{3}$ Centre for Theoretical Chemistry and Physics, The New Zealand Institute for Advanced Study, \\ Massey University Auckland, Private Bag 102904, 0745 Auckland, New Zealand \\ ${ }^{4}$ School of Chemistry, Tel Aviv University, 69978 Tel Aviv, Israel \\ ${ }^{5}$ Physics Department, University of Nevada, Reno, Nevada 89557, USA \\ ${ }^{6}$ Physikalisch-Technische Bundesanstalt, Bundesallee 100, 38116 Braunschweig, Germany \\ ${ }^{7}$ Institut für Quantenoptik, Leibniz Universität Hannover, \\ Welfengarten 1, 30167 Hannover, Germany \\ (Received 9 January 2015; published 16 April 2015)
}

\begin{abstract}
We measure optical spectra of Nd-like W, Re, Os, Ir, and Pt ions of particular interest for studies of a possibly varying fine-structure constant. Exploiting characteristic energy scalings we identify the strongest lines, confirm the predicted $5 s-4 f$ level crossing, and benchmark advanced calculations. We infer two possible values for optical $M 2 / E 3$ and $E 1$ transitions in $\operatorname{Ir}^{17+}$ that have the highest predicted sensitivity to a variation of the fine-structure constant among stable atomic systems. Furthermore, we determine the energies of proposed frequency standards in $\mathrm{Hf}^{12+}$ and $\mathrm{W}^{14+}$.
\end{abstract}

DOI: 10.1103/PhysRevLett.114.150801

PACS numbers: 06.20.Jr, 31.15.am, 31.15.bw, 32.30.Jc

Highly charged ions (HCIs) are currently in the focus of theoretical studies analyzing their applications to frequency metrology and tests of a variation of the fine-structure constant $\alpha$ [1-12]. In most of the proposed HCIs with atomic number $Z=55-98$ and in charge states from 7 to 35 , the complex electronic structures are experimentally unknown, and accurate calculations are extremely difficult. In view of novel techniques for sympathetically cooling HCIs in Paul traps [13-15] aiming at quantum logic spectroscopy on highly forbidden transitions [16], such data are urgently required.

Observations from quasar absorption spectra have suggested a spatial variation of the value of the fine-structure constant $\alpha$ over cosmological dimensions [17], characterized by a dipolar distribution with a value of $10^{-6} \mathrm{GLyr}^{-1}$. Laboratory experiments [18-21] have not yet reached the accuracy needed to test this dipolar pattern, which translates to a temporal variation of $10^{-19} \mathrm{yr}^{-1}$ [22] due to the motion of the Earth. Future optical clocks based on HCIs [3] could improve such tests. Interconfiguration transitions in HCIs have a high sensitivity to a variation of $\alpha$ due to large relativistic contributions to their binding energies. Advantageously, they have a strongly suppressed sensitivity to external perturbations [12]. However, interconfiguration transitions quickly shift from the optical laser range into the extreme ultraviolet or $\mathrm{x}$-ray region with increasing charge state [23]. Nonetheless, at level crossings with two or more nearly degenerate electronic configurations, forbidden, and thus narrow, optical transitions with an enhanced sensitivity $[1,2]$ arise. In particular, the Nd-like system $\operatorname{Ir}^{17+}$ offers narrow lines between three electronic configurations $4 f^{14}, 4 f^{13} 5 s^{1}$, and $4 f^{12} 5 s^{2}$ with the highest ever predicted sensitivity in a stable atomic system [2]. However, calculations for this system are exceptionally difficult, and the predicted energies for intraconfiguration $M 1$ transitions ideally suited for clock applications [6] can exhibit errors on the $10 \%$ level, as can be seen below. For interconfiguration transitions, uncertainties at the $\mathrm{eV}$ level are expected. Thus, optical line identification becomes extremely difficult.

In this Letter, we demonstrate a method to reliably identify transitions in such complex systems. It employs the basic laws governing atomic fine-structure splitting, which predict smooth energy scaling of the $M 1$ transitions as a function of the atomic number $Z$ (see Fig. 1). The method is advantageous for a growing number of proposed applications $[1-9,11]$. We stringently test the results by an independent identification based on resolved Zeeman splittings in the strong magnetic field of an electron beam ion trap (EBIT). Armed with these identifications, we could confirm the predicted $5 s-4 f$ level crossing and benchmark our own atomic structure calculations.

The experiment was performed with the Heidelberg EBIT [24]. Atoms injected into the EBIT are sequentially ionized to the desired charge state and subsequently excited by a monoenergetic electron beam focused to 

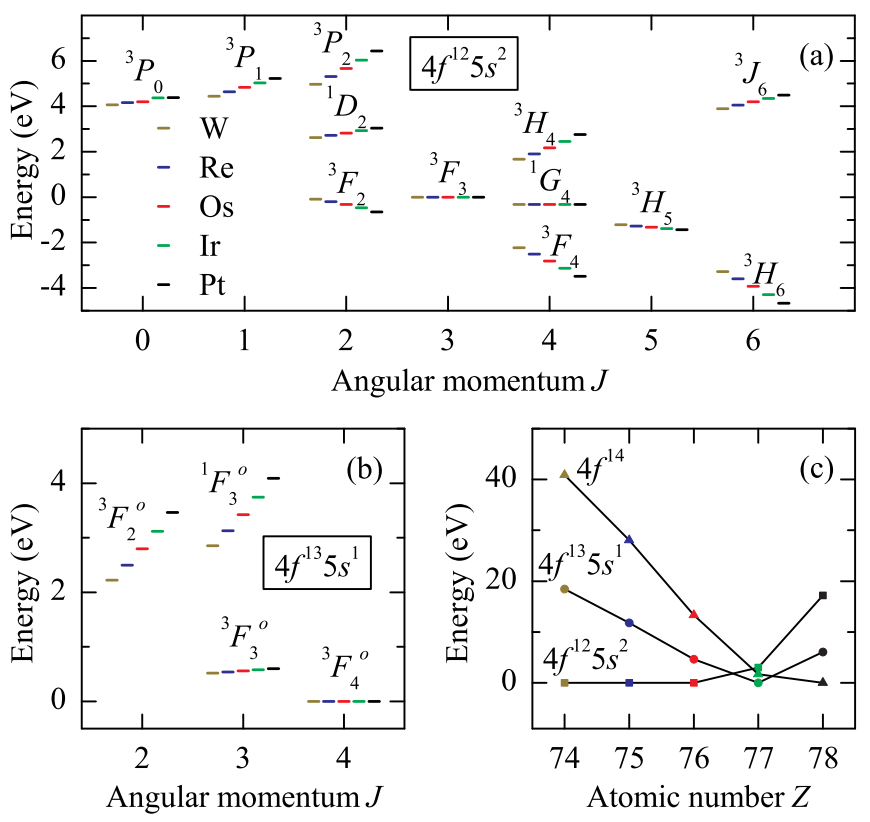

FIG. 1 (color online). Grotrian diagrams based on our Fock space coupled cluster calculations of the (a) $4 f^{12} 5 s^{2}$, and (b) $4 f^{13} 5 s^{1}$ configurations of Nd-like $\mathrm{W}, \mathrm{Re}, \mathrm{Os}$, Ir, and $\mathrm{Pt}$ $(Z=74-78)$. A smooth energy scaling depending on the atomic number, shown here relative to the levels (a) ${ }^{3} F_{3}$ and (b) ${ }^{3} F_{4}^{o}$, is observable. In (c), the energy separation between the lowest energy states in each configuration, $4 f^{14}{ }^{1} S_{0}, 4 f^{13} 5 s^{13} F_{4}^{o}$, and $4 f^{12} 5 s^{2} H_{6}$, is plotted. The three configurations cross near $\operatorname{Ir}^{17+}$ resulting in several $\alpha$-sensitive optical transitions in this species.

approximately $50 \mu \mathrm{m}$ diameter by superconducting Helmholtz coils. These ions are axially trapped in a potential well formed by the surrounding drift tubes and radially confined by the negative space-charge potential of the electron beam. Moderate currents in the range of 10-40 $\mathrm{mA}$ were used to avoid excessive ion heating due to electron collisions. Scanning the electron beam energy enables the assignment of lines to specific charge states (see Refs. $[25,26]$ and references therein). We tuned the electron beam energy in the range of $170-900 \mathrm{eV}$ to maximize the signal of the required charge state. The ions are collisionally excited to a dense manifold of levels close to the continuum and cascade down to the ground state, emitting photons ranging from the VUV to the visible range. We studied the $\mathrm{Nd}$-like isoelectronic ions $\mathrm{W}^{14+}, \mathrm{Re}^{15+}$, $\mathrm{Os}^{16+}$, $\operatorname{Ir}^{17+}$, and $\mathrm{Pt}^{18+}(Z=74-78)$ in the optical region spanning 250-650 $\mathrm{nm}$. To facilitate charge state identification, spectroscopy in the extreme-ultraviolet domain was also performed [26].

To obtain spectra, the trapped ion cloud is imaged onto the entrance slit of a Czerny-Turner spectrometer. For overview spectra, a 300 lines $/ \mathrm{mm}$ grating provided a resolving power of 1250 . The brightest spectral lines of Ir were additionally acquired with a 2400 lines $/ \mathrm{mm}$ grating, reaching a resolving power of 30000 , sufficient to separate the Zeeman components split by the $8 \mathrm{~T}$ magnetic field of the EBIT. Spectra were recorded with a CCD having $2048 \times 512$ pixels, a size of $30 \times 8 \mathrm{~mm}^{2}$, and cooled with liquid nitrogen [thermal noise 1 $e^{-} /($pixel $h)$, readout noise $3 e^{-} /$pixel]. We corrected the images for astigmatism and coma and removed cosmic ray events before integrating over the nondispersive axis of the CCD chip to generate spectra. Calibration spectra were obtained using hollow-cathode spectral lamps, as in earlier work $[27,28]$. The final wavelength uncertainties are estimated to be $0.02 \mathrm{~nm}$ for the 300 lines $/ \mathrm{mm}$ grating and $10^{-4}-10^{-3} \mathrm{~nm}$ for the 2400 lines $/ \mathrm{mm}$ grating.

Between 9 and 30 transitions for each element and charge state were analyzed [29]. For the five elements under investigation, we expect a smooth energy dependence on $Z$ for each transition (indexed with subscript $i$, see Fig. 1). First, these theoretical energy scalings are parametrized by Taylor expansions of the form

$$
E_{i}(Z)=A_{i}+B_{i}\left(Z-Z_{\text {mean }}\right)+C_{i}\left(Z-Z_{\text {mean }}\right)^{2}
$$

describing this behavior sufficiently well. Second, an algorithm automatically searches the experimental spectra for such scalings using all possible combinations of measured transition energies, with $A_{i}, B_{i}$, and $C_{i}$ as fit parameters. The scaling functions are expanded around the central Os ${ }^{16+}$ spectrum using $Z_{\text {mean }}=76$. A comparison between the experimental fits and the parameters extracted from relativistic multireference Fock space coupled cluster (FSCC) calculations [37,38], performed using the TRAFS3C atomic code [39], is shown in Fig. 2(b). On the basis of the theoretical predictions, we enforce a maximum fit residual given by a standard deviation $\leq 1 \times 10^{-3} \mathrm{eV}$ on experimental scalings. Additionally, only linear terms with $0.05 \mathrm{eV} / Z \leq B_{i} \leq 0.35 \mathrm{eV} / Z$ and quadratic ones with $0.0001 \mathrm{eV} / Z^{2} \leq C_{i} \leq 0.02 \mathrm{eV} / Z^{2}$ are considered. A Monte Carlo method was applied for estimating the number of expected false positives by randomly redistributing the spectral lines in each measured spectrum, yielding a probability below 5\% for finding even a single false positive. As shown in Fig. 2(b), the combined information derived from the fitted coefficients and the corresponding curves reveals the underlying isoelectronic transitions. Third, by comparing the experimental $\left(A_{i}, B_{i}, C_{i}\right)$ parameters with their theoretical counterparts and minimizing the associated $\chi^{2}$, a clear identification of the specific levels involved in the transitions was achieved. We estimate that the transition energies of the neighboring isoelectronic ions can be extrapolated with an uncertainty of a few meV. Besides the $Z$ scaling of the transition energies, the complexity of changes in the line intensity patterns gives additional confidence that level crossings do occur.

An immediate application is the transition ${ }^{3} F_{4}{ }^{3} H_{6}$ in Nd-like $\mathrm{Hf}^{12+}$ and $\mathrm{W}^{14+}$, suggested as a frequency standard with radiative width on the order of $\mu \mathrm{Hz}$ and potential relative systematic uncertainties of less than $10^{-19}$ [6]. For 


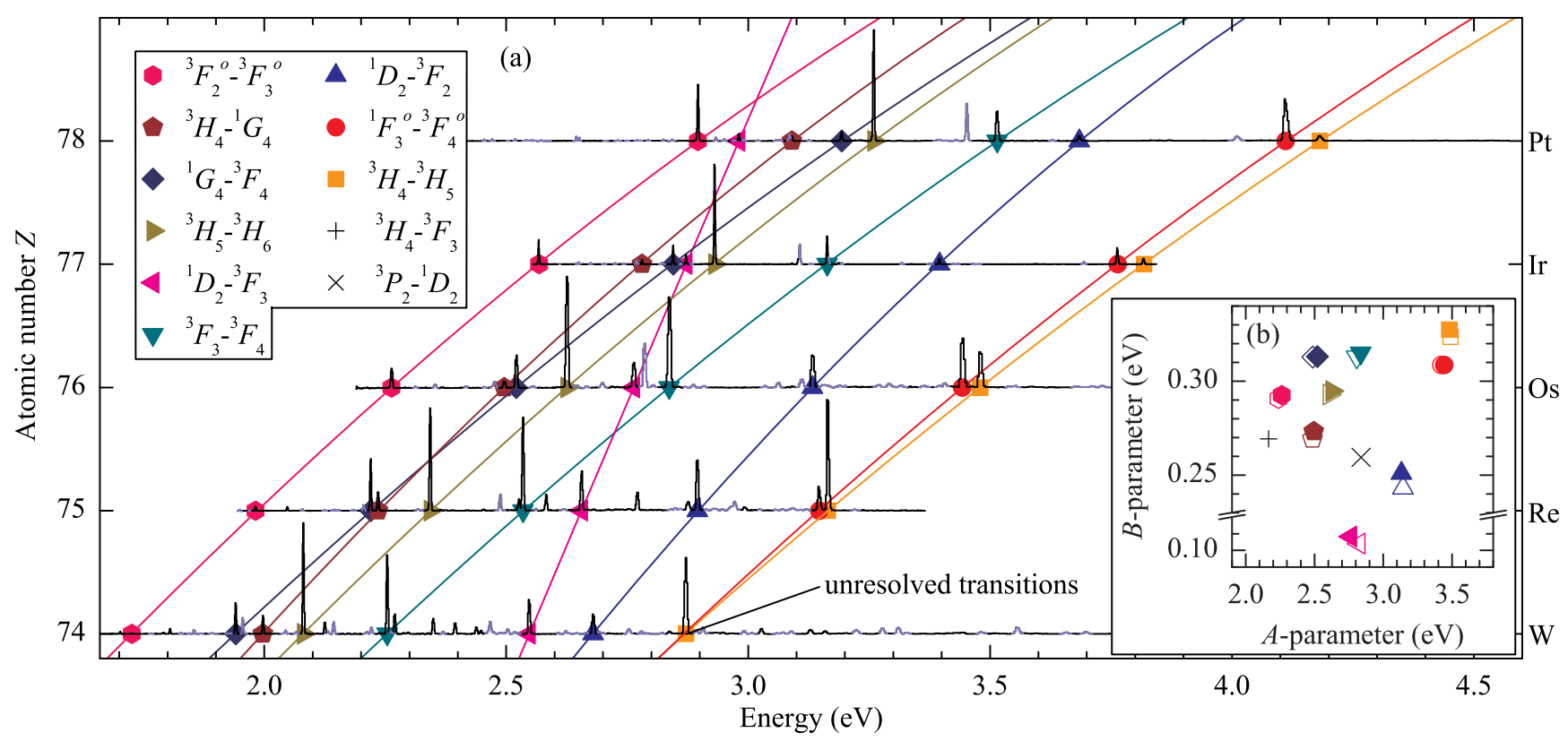

FIG. 2 (color online). Identification of transitions by their characteristic energy scaling for Nd-like W, Re, Os, Ir, and Pt ions. In (a), their measured optical spectra (black lines) are shown; transitions belonging to other charge states are depicted in gray. Several transition energies $E_{i}$ (symbols) are found to follow a function given by Eq. (1) (colored lines). A comparison between the fitted offsets $A_{i}$ and the linear coefficients $B_{i}$ (filled symbols) and the corresponding parameters predicted by Fock space coupled cluster calculations (open symbols) is shown in (b). Uncertainties on the fitted parameters $A_{i}$ and $B_{i}$ are smaller than the symbol. Not all possible transitions could be observed and two theoretically expected scalings (black crosses) have not been identified at the current sensitivity. Through a $\chi^{2}$ minimization of theoretical and experimental $\left(A_{i}, B_{i}, C_{i}\right)$-parameter assignments, spectral lines along the measured isoelectronic sequence can be clearly identified with their transitions.

$\mathrm{W}^{14+}$, we infer (using ${ }^{3} \mathrm{~F}_{4^{-}}{ }^{1} G_{4^{-}}{ }^{3} H_{4^{-}}{ }^{3} H_{5}{ }^{-3} H_{6}$ ) its value to $1.0140(2) \mathrm{eV}$ (from Ref. [6], $1.141 \mathrm{eV}$ ). By following the M1 scaling functions for these levels, we extrapolate the energy of the same transition in $\mathrm{Hf}^{12+}$ to $0.934(6) \mathrm{eV}$ (from [6]: $1.062 \mathrm{eV}$ ).

We tested these identifications using high-resolution spectra of the brightest $\operatorname{Ir}^{17+}$ spectral lines. The $\operatorname{Ir}^{17+}$ line shapes are compared to a model using the $g$ factors $g\left(g^{\prime}\right)$ of the upper (lower) levels calculated with the AMBIT code [40]. The relative amplitudes were obtained through squared Clebsch-Gordan coefficients $\left(C_{J m_{J} 1 \Delta m}^{J^{\prime} m_{J}+\Delta m}\right)^{2}$, where $J\left(J^{\prime}\right)$ is the upper (lower) level total angular momentum, $m_{J}$ is the upper level magnetic quantum number, and $\Delta m$ is the change of $m_{J}$. The theoretical line patterns are convolved with a Gaussian function yielding

$$
\begin{aligned}
f(E)= & \sum_{\Delta m} a_{\Delta m} \sum_{m_{J}}\left(C_{J m_{J} 1 \Delta m}^{J^{\prime} m_{J}+\Delta m}\right)^{2} \\
& \times \exp \left[-\frac{\left(E-E_{0}-\mu_{B} B\left[m_{J}\left(g-g^{\prime}\right)+\Delta m g^{\prime}\right]\right)^{2}}{2 w^{2}}\right]
\end{aligned}
$$

as a function of energy $E$, with $E_{0}$ being the center of the spectral line. The separation of the Zeeman components is proportional to the 8.000(5) T magnetic field $B$. The three fit parameters are the amplitudes for the different polarizations $a_{-1}=a_{1}$ and $a_{0}$, taking into account the signal strength along the direction of observation assuming equally populated excited magnetic substates, and a common width $w$ resulting from Doppler broadening and instrumental resolution [28]. The excellent agreement, within the expected error of the calculated $\left(g, g^{\prime}\right)$ factors, between fit and experimentally observed spectrum shown in Fig. 3 validates the $M 1$ scaling identification.

Optical transitions in Nd-like systems near level-crossing degeneracies represent a stringent benchmark for atomic structure calculations of strongly correlated many-electron HCIs. Figure 4 shows a comparison between our experimental results and different $a b$ initio calculations, four of which are part of this work. We find that FSCC calculations are the most accurate, with a standard deviation of only $0.03 \mathrm{eV}$. This experimental outcome proves their suitability for the reliable identification of most observed transitions, even in complicated cases like the present one.

Employing Ritz's combination principle with three transitions (see Fig. 1), using the known ${ }^{1} D_{2}{ }^{-3} F_{2} M 1$ transition, we assigned the transitions ${ }^{3} P_{1}-{ }^{3} F_{2}=$ $5.4691(5) \mathrm{eV}$ and ${ }^{3} P_{1}{ }^{-1} D_{2}=2.07395(7) \mathrm{eV}$, in very good agreement with FSCC predictions. In order to find further assignments, we use combinations of four transitions. 


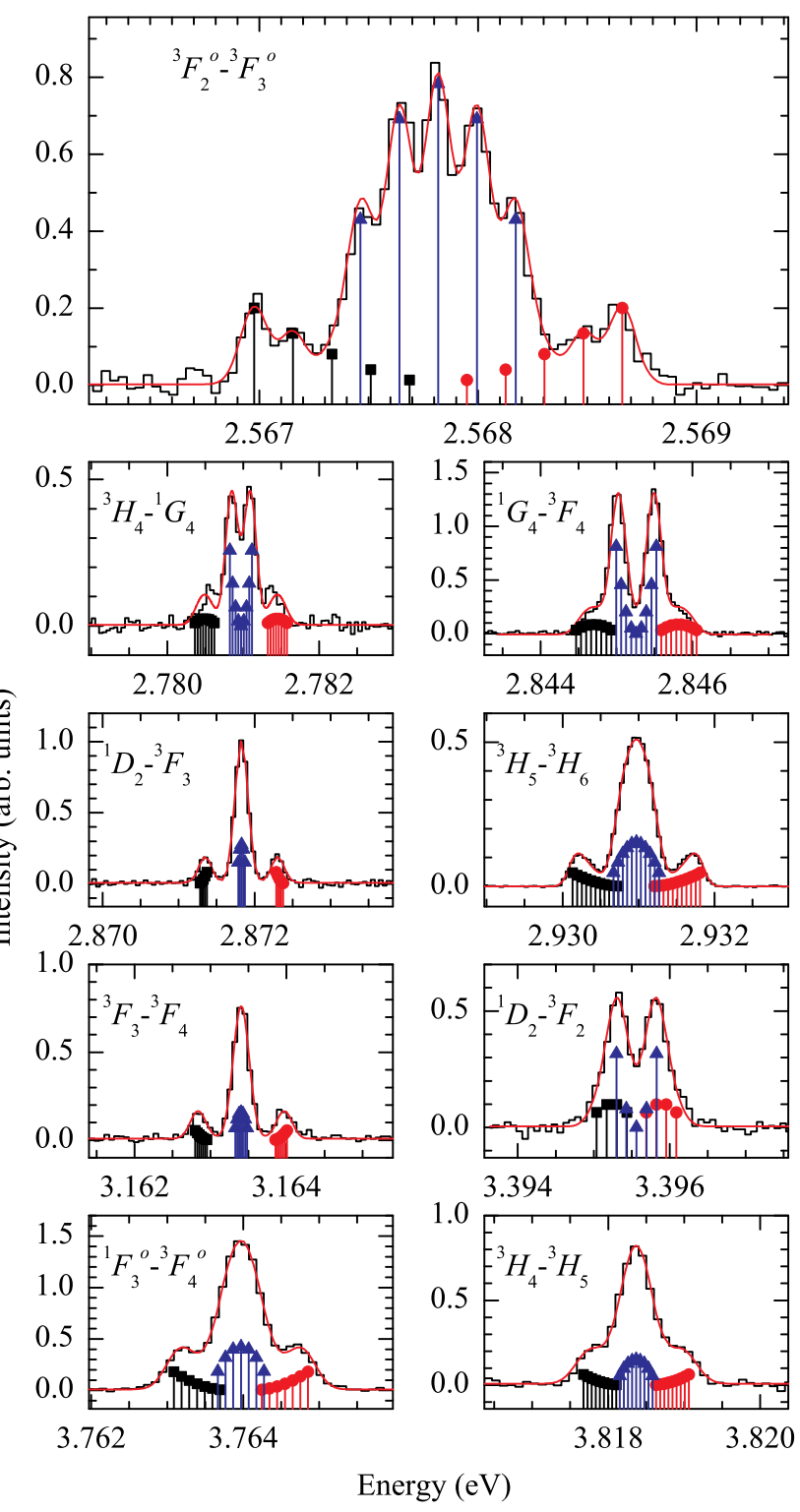

FIG. 3 (color online). Identification of highly resolved $\mathrm{Ir}^{17+}$ spectral lines between odd(even)-parity states belonging to the $4 f^{13} 5 s^{1}\left(4 f^{12} 5 s^{2}\right)$ configurations using their Zeeman components. Their characteristic splitting (measured, black lines) is fitted with a model function [red line, see Eq. (2)] using calculated Zeeman components (black squares: $\Delta m=+1$, blue triangles: $\Delta m=0$, red circles: $\Delta m=-1$ ).

Interestingly, this leads to two possible scenarios including two weak $E 1$ interconfiguration lines: ${ }^{3} F_{4}{ }^{3} F_{4}^{o}=$ $5.1623(4) \mathrm{eV}$ and ${ }^{3} F_{3^{-}}{ }^{1} F_{3}^{o}=4.5619(3) \mathrm{eV}$ (using $\left.{ }^{3} F_{3}{ }^{3} F_{4}-{ }^{3} F_{4}^{o}{ }^{1} F_{3}^{o}\right) \quad$ or $\quad{ }^{3} F_{3^{-}}{ }^{-1} F_{3}^{o}=5.1623(4) \mathrm{eV} \quad$ and ${ }^{1} G_{4^{-}}{ }^{1} F_{3}^{o}=4.8441(4) \mathrm{eV}$ (using ${ }^{3} F_{3}{ }^{-3} F_{4^{-}}{ }^{1} G_{4^{-}}{ }^{1} F_{3}^{o}$ ). With this result, the closeness of the two configurations $4 f^{13} 5 s$ and $4 f^{12} 5 s^{2}$ is proven. This discovery paves the way for numerous important applications of HCI. We can further infer two possible values for the sought-after $M 2 / E 3$ transition: ${ }^{3} H_{6}{ }^{3} F_{4}^{o}=4.0392(4)$ or $4.6397(4) \mathrm{eV}$, respectively. Our CIDFS calculations favor the first candidate,

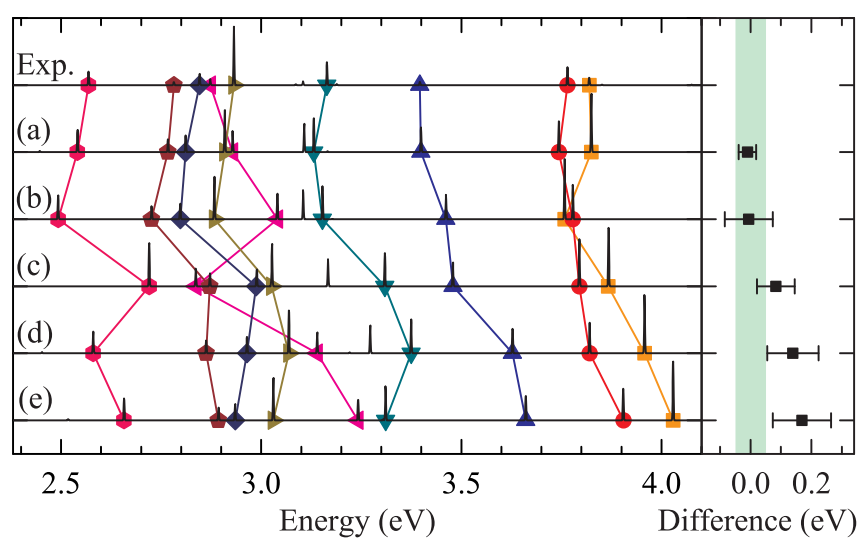

FIG. 4 (color online). Left: Comparison between the experimental spectrum of the Nd-like $\mathrm{Ir}^{17+}$ ion and different atomic calculations performed in this work [except (e)] using the same symbols and colors for the transitions as in Fig. 2. For theories, only $M 1$ transitions in the $4 f^{12} 5 s^{2}$ and $4 f^{13} 5 s^{1}$ configuration are displayed, using Einstein coefficients as amplitudes. (a) Fock space coupled cluster, (b) configuration interaction Dirac-Fock Sturmian (CIDFS) [41,42], (c) COWAN code [43], (d) Flexible Atomic Code (FAC) [44], (e) configuration interaction $(\mathrm{CI})[2,45]$. Einstein coefficients were not available for the FSCC and CI methods; the FAC ones were used instead. The identified $M 1$ transitions (symbols) in the data are connected across the spectra. Right: Mean difference (symbols) and standard deviation (error bars) between theories and experiment. The average separation between the measured transitions of $0.1 \mathrm{eV}$ is indicated with the green band.

while our CI calculations favor the second one. The former agree better in general with the observed $M 1$ transitions. However, we do not have sufficient evidence to claim the same for $E 1$ transitions. According to a Monte Carlo estimation, the a priori chance is $16 \%$ to draw a single false positive and for two false positives it is a few percent. Both mutually exclusive scenarios have similar likelihoods. The weakness of the $E 1$ transitions compared to the bright $M 1$ lines is supported by our calculations showing that the $E 1$-matrix elements between the intervening configurations are very small. However, the sensitivity and accuracy of future measurements could easily be enhanced, and further Ritz combinations may be uncovered, removing the present ambiguity.

In conclusion, our spectroscopic study along the Nd-like isoelectronic sequence of $\mathrm{W}^{14+}, \mathrm{Re}^{15+}, \mathrm{Os}^{16+}, \mathrm{Ir}^{17+}$, and $\mathrm{Pt}^{18+}$ has encompassed and revealed the $5 s-4 f$ level crossing. Exploiting the fact that $M 1$ fine-structure transitions smoothly scale with $Z$, we have demonstrated a straightforward, reliable, and general method of spectral line assignments by the isoelectronic scaling of transitions (LINE ASSIST), with applications also to many other cases of lesser or higher complexity. We have chosen prime examples with promising proposed applications and thus far untested theory. High-resolution measurements of the nine brightest lines in $\mathrm{Ir}^{17+}$ revealed their Zeeman splitting, and 
its analysis enabled independent identifications in perfect agreement with the scaling method. In total, 85 spectral lines were measured in Nd-like ions, of which 47 were assigned to $M 1$ transitions. Here, with dedicated FSCC calculations, we have attained for this type of systems an at least 3 times better accuracy within an electronic configuration than other available advanced atomic structure methods. This is probably due to an effective treatment of the here-relevant electron correlations. Crucially, these $M 1$ identifications and a few $E 1$ identifications in $\operatorname{Ir}^{17+}$ lead to the inference of two possible values for the energy of the uniquely $\alpha$-sensitive and narrow $M 2 / E 3$ transition in this ion, a line with particularly interesting properties according to recent systematic theoretical studies. Furthermore, the transition energies of proposed optical clocks in $\mathrm{Hf}^{12+}$ and $\mathrm{W}^{14+}$ were obtained. These steps enable follow-up laser spectroscopic studies suitable for optical clock applications. For this purpose, we have recently commissioned an experiment in which HCIs were extracted from an EBIT into a novel cryogenic Paul trap [13] where they were sympathetically laser cooled into Coulomb crystals at temperatures of few $\mathrm{mK}$ [46], thus preparing HCIs for quantum-logic readout schemes similar to that of Ref. [16].

*Corresponding author.

alexander.windberger@mpi-hd.mpg.de

${ }^{\dagger}$ Corresponding author.

crespojr@mpi-hd.mpg.de

"Corresponding author.

O.Versolato@arcnl.nl

Present address: Advanced Research Center for Nanolithography, Science Park 110, 1098 XG Amsterdam, The Netherlands.

[1] J. C. Berengut, V. A. Dzuba, and V. V. Flambaum, Phys. Rev. Lett. 105, 120801 (2010).

[2] J. C. Berengut, V. A. Dzuba, V. V. Flambaum, and A. Ong, Phys. Rev. Lett. 106, 210802 (2011).

[3] J. C. Berengut, V. A. Dzuba, V. V. Flambaum, and A. Ong, Phys. Rev. A 86, 022517 (2012).

[4] J. C. Berengut, V. A. Dzuba, V. V. Flambaum, and A. Ong, Phys. Rev. Lett. 109, 070802 (2012).

[5] A. Derevianko, V. A. Dzuba, and V. V. Flambaum, Phys. Rev. Lett. 109, 180801 (2012).

[6] V. A. Dzuba, A. Derevianko, and V. V. Flambaum, Phys. Rev. A 86, 054501 (2012).

[7] V. A. Dzuba, V. V. Flambaum, and H. Katori, Phys. Rev. A 91, 022119 (2015).

[8] M. S. Safronova, V. A. Dzuba, V. V. Flambaum, U. I. Safronova, S. G. Porsev, and M. G. Kozlov, Phys. Rev. A 90, 042513 (2014).

[9] M. S. Safronova, V. A. Dzuba, V. V. Flambaum, U. I. Safronova, S. G. Porsev, and M. G. Kozlov, Phys. Rev. A 90, 052509 (2014).

[10] M. S. Safronova, V. A. Dzuba, V. V. Flambaum, U. I. Safronova, S. G. Porsev, and M. G. Kozlov, Phys. Rev. Lett. 113, 030801 (2014).
[11] V. I. Yudin, A. V. Taichenachev, and A. Derevianko, Phys. Rev. Lett. 113, 233003 (2014).

[12] S. Schiller, Phys. Rev. Lett. 98, 180801 (2007).

[13] M. Schwarz, O. O. Versolato, A. Windberger, F. R. Brunner, T. Ballance, S. N. Eberle, J. Ullrich, P. O. Schmidt, A. K. Hansen, A. D. Gingell, M. Drewsen, and J. R. Crespo López-Urrutia, Rev. Sci. Instrum. 83, 083115 (2012).

[14] O. O. Versolato, M. Schwarz, A. Windberger, J. Ullrich, P. O. Schmidt, M. Drewsen, and J. R. Crespo López-Urrutia, Hyperfine Interact. 214, 189 (2013).

[15] A. Windberger, M. Schwarz, O. O. Versolato, T. Baumann, H. Bekker, L. Schmöger, A. K. Hansen, A. D. Gingell, L. Klosowski, S. Kristensen, P. O. Schmidt, J. Ullrich, M. Drewsen, and J. R. Crespo López-Urrutia, AIP Conf. Proc. 1521, 250 (2013).

[16] P. O. Schmidt, T. Rosenband, C. Langer, W. M. Itano, J. C. Bergquist, and D. J. Wineland, Science 309, 749 (2005).

[17] J. K. Webb, J. A. King, M. T. Murphy, V. V. Flambaum, R. F. Carswell, and M. B. Bainbridge, Phys. Rev. Lett. 107, 191101 (2011).

[18] T. Rosenband, D. B. Hume, P. O. Schmidt, C. W. Chou, A. Brusch, L. Lorini, W. H. Oskay, R. E. Drullinger, T. M. Fortier, J. E. Stalnaker, S. A. Diddams, W. C. Swann, N. R. Newbury, W. M. Itano, D. J. Wineland, and J. C. Bergquist, Science 319, 1808 (2008).

[19] N. Leefer, C. T. M. Weber, A. Cingöz, J. R. Torgerson, and D. Budker, Phys. Rev. Lett. 111, 060801 (2013).

[20] R. M. Godun, P. B. R. Nisbet-Jones, J. M. Jones, S. A. King, L. A. M. Johnson, H. S. Margolis, K. Szymaniec, S. N. Lea, K. Bongs, and P. Gill, Phys. Rev. Lett. 113, 210801 (2014).

[21] N. Huntemann, B. Lipphardt, C. Tamm, V. Gerginov, S. Weyers, and E. Peik, Phys. Rev. Lett. 113, 210802 (2014).

[22] J. C. Berengut and V. V. Flambaum, Europhys. Lett. 97, 20006 (2012)

[23] J. D. Gillaspy, J. Phys. B 34, R93 (2001).

[24] J. R. Crespo López-Urrutia, A. Dorn, R. Moshammer, and J. Ullrich, Phys. Scr. T80, 502 (1999).

[25] J. R. Crespo López-Urrutia, P. Beiersdorfer, K. Widmann, and V. Decaux, Can. J. Phys. 80, 1687 (2002).

[26] H. Bekker, O. O. Versolato, A. Windberger, N. S. Oreshkina, R. Schupp, T. M. Baumann, Z. Harman, C. H. Keitel, P. O. Schmidt, J. Ullrich, and J. R. Crespo López-Urrutia, J. Phys. B. (to be published).

[27] I. Draganić, J. R. Crespo López-Urrutia, R. DuBois, S. Fritzsche, V. M. Shabaev, R. Soria Orts, I. I. Tupitsyn, Y. Zou, and J. Ullrich, Phys. Rev. Lett. 91, 183001 (2003).

[28] R. Soria Orts, Z. Harman, J. R. Crespo López-Urrutia, A. N. Artemyev, H. Bruhns, A. J. González Martínez, U. D. Jentschura, C. H. Keitel, A. Lapierre, V. Mironov, V. M. Shabaev, H. Tawara, I. I. Tupitsyn, J. Ullrich, and A. V. Volotka, Phys. Rev. Lett. 97, 103002 (2006).

[29] See the Supplemental Material at http://link.aps.org/ supplemental/10.1103/PhysRevLett.114.150801, for underlying data and magnified spectra, which includes Refs. [30-36].

[30] J. Sucher, Phys. Rev. A 22, 348 (1980).

[31] Y. Ishikawa, R. Baretty, and R. C. Binning, Jr., Chem. Phys. Lett. 121, 130 (1985). 
[32] I. Lindgren, Nucl. Instrum. Methods Phys. Res., Sect. B 31, 102 (1988).

[33] I. Lindgren, in Many-Body Methods in Quantum Chemistry, edited by U. Kaldor, Lecture Notes in Chemistry 52, 293 (1989).

[34] G. L. Malli, A. B. F. Da Silva, and Y. Ishikawa, Phys. Rev. A 47, 143 (1993).

[35] E. R. Peck and K. Reeder, J. Opt. Soc. Am. 62, 958 (1972).

[36] A. Kramida, Y. Ralchenko, J. Reader, and NIST ASD Team, NIST Atomic Spectra Database, version 5.2, 2014 (retrieved 08/01/2015)

[37] D. Mukherjee and S. Pal, Adv. Quantum Chem. 20, 291 (1989).

[38] U. Kaldor and E. Eliav, Adv. Quantum Chem. 31, 313 (1998).

[39] Tel-Aviv Relativistic Atomic Fock Space coupled cluster code, written by E. Eliav, U. Kaldor, and Y. Ishikawa (1990).
[40] J. C. Berengut, V. V. Flambaum, and M. G. Kozlov, Phys. Rev. A 73, 012504 (2006).

[41] I. I. Tupitsyn, V. M. Shabaev, J. R. Crespo López-Urrutia, I. Draganić, R. Soria Orts, and J. Ullrich, Phys. Rev. A 68, 022511 (2003).

[42] I. I. Tupitsyn, A. V. Volotka, D. A. Glazov, V. M. Shabaev, G. Plunien, J. R. Crespo López-Urrutia, A. Lapierre, and J. Ullrich, Phys. Rev. A 72, 062503 (2005).

[43] R. D. Cowan, The Theory of Atomic Structure and Spectra (University of California Press, Berkeley, CA, 1981), Vol. 3.

[44] M. F. Gu, Can. J. Phys. 86, 675 (2008).

[45] V. A. Dzuba, V. V. Flambaum, and J. K. Webb, Phys. Rev. A 59, 230 (1999).

[46] L. Schmöger, O. O. Versolato, M. Schwarz, M. Kohnen, A. Windberger, B. Piest, S. Feuchtenbeiner, J. PedregosaGutierrez, T. Leopold, P. Micke, A. K. Hansen, T. M. Baumann, M. Drewsen, J. Ullrich, P. O. Schmidt, and J. R. Crespo López-Urrutia, Science 347, 1233 (2015). 